\title{
A Hybrid Estimation Theoretic-POCS Method for Tomographic Image Reconstruction
}

\author{
Fernando V. Salina ${ }^{1}$, Nelson D. A. Mascarenhas ${ }^{2}$ \\ ${ }^{1}$ USP - Universidade de São Paulo, Caixa Postal 369, 13560-970 São Carlos, SP, Brasil \\ salina@if.sc.usp.br \\ ${ }^{2}$ UFSCar - Universidade Federal de São Carlos, Cx P 676, 13565-905 São Carlos- SP, Brasil \\ nelson@dc.ufscar.br
}

\begin{abstract}
We present in this work a new method for the tomographic reconstruction with Poisson noise corrupted projections. The reconstruction method is a hybrid estimation theoretic-POCS (Projection Onto Convex Sets) method performed by first estimating the noisy projections using Anscombe Transformation, Discrete Wavelet Transform and pointwise Wiener filter and subsequently reconstructing the images through a parallel POCS method. The use of a previous filtering of the projections imposes a tighter restriction on the observation of the POCS method, allowing a better reconstruction, with a very little increase in computational cost.
\end{abstract}

\section{Introduction}

In several problems in image processing, as tomographic reconstruction, observations are often modeled as a noisy signal. The most common assumption in these models is that the noise is modeled as conditionally independent Gaussian random variables. The noise is also usually modeled as stationary independent zero-mean Gaussian process. However, in many problems of physics, the recorded data are not modeled by Gaussian noise but as the realization of a Poisson process.

In tomographic reconstruction of images, the corruption of the measurements by Poisson noise occurs very often with nuclear radiation low level of the counting process that is involved. The exposure time of a body to the radiation is directly linked to the photons count obtained by the tomograph scanner. With low photons count, very noisy projections are produced, that can result in reconstructed images with unacceptable amount of noise. In previous works, Mascarenhas et al [1,2] have proposed a method that kept a compromisse between purely statistical methods and convolution-backprojection techniques. These methods are implemented by first filtering the Poisson noise of the projections, followed by the use of algorithms associated with convolution-backprojection.

Methods of tomographic reconstruction based on POCS (Projections onto Convex Sets) consider restrictions over the set of possible solutions. Besides allowing the incorporation of a priori knowlegde, through convex restrictions, the method of projections allows the tomographic reconstruction to be independent of the source-detector geometry used by the tomograph scanner. These methods are presented and discussed in [3]. The parallel POCS method obtains better results than the sequential POCS in the presence of noise because in the case where there is no intersection of the restriction sets, the parallel method provides a solution while the sequential methods cycle through the solutions [3].

The objective of this work is to compare the method of reconstruction based on parallel POCS by first filtering the projections with that based on a pure POCS approach.

The paper is organized as follows. In Section 2 we present a brief introduction to the theory of POCS methods and describe sequential and parallel POCS algorithms. In Section 3 a method for wavelet denoising and local Wiener filtering is presented. The tomographic reconstruction method is presented in Section 4 and Section 5 provides results on experimental data. The results are discussed in Section 6.

\section{POCS Algorithm}

Many problems can be described under the convex restriction sets form [4]. The solution for these problems satisfies all the imposed restrictions, that is, the search of the wanted solution consists in finding a point belonging to the intersection of all the sets.

If we suppose that there exist $n$ restriction sets, represented by $C_{i}(i=1,2, \ldots, n)$, the solution to the problem is in the intersection of the sets, represented by Eq. (1). 
$C_{0}=\bigcap_{i=0}^{n} C_{i}$

If the sets $C_{i}(i=1,2, \ldots, n)$ are closed and convex and their intersection $C_{0}$ is non-empty, the successive projections on the sets will converge to a vector which belongs to $C_{0}$. Equation (2) represents the algorithm, assuming that $x_{0}$ is any point and represents the initial estimate, and $P_{C}$ is a projection operator in $\mathrm{C}$.

$x_{k+1}=P_{C_{n}} \cdots P_{C_{2}} P_{C_{1}} x_{k}$

Youla [9] demonstrated that, if $C_{0}$ is non empty, for each $x \in H$, where $H$ represents a Hilbert space, the sequence given by (2) converges weakly to a point in $C_{0}$. Although, theoretically, this convergence is weak, in practice the convergence to $C_{0}$ is strong. Figure 1 shows the graphic representation of that convergence.

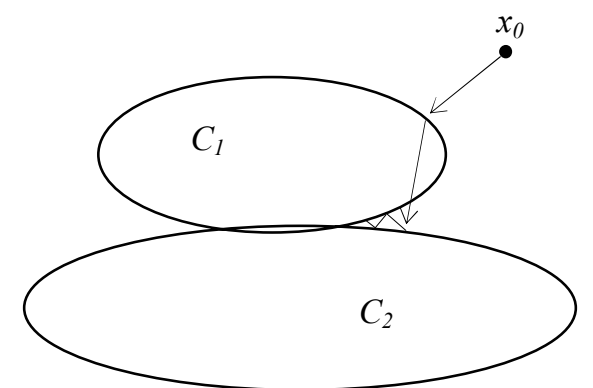

Figure 1: Geometrical representation of the POCS sequential method convergence

When there is not an intersection of the restriction sets, the POCS sequential algorithm does not converge to a point and remains oscillating among the restrictions sets, as shown in Figure 2.

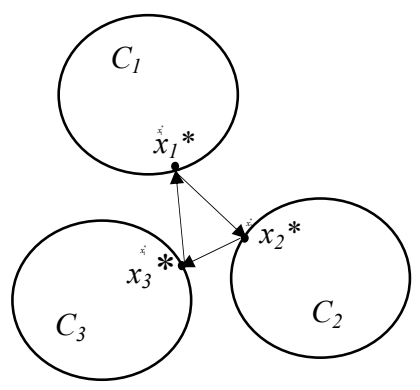

Figure 2: Algorithm behavior of a sequential POCS when there is no intersection of the convex sets

\subsection{Parallel POCS Algorithm}

The POCS algorithm can be implemented in parallel. In this implementation, the vector is projected on all sets simultaneously and to each projection on a set a weight is assigned. Eq. (3) describes the parallel POCS algorithm.

$$
\begin{aligned}
& x_{k+1}=x_{k}+\sum_{i=1}^{n} w_{i}\left(P_{C_{i}}-x_{k}\right) \quad k=0,1,2,3, \cdots \\
& \sum_{i=1}^{n} w_{i}=1
\end{aligned}
$$

Observe that the summation of all weights is equal to one, according to Eq. (4).

If there is not intersection of the restriction sets, the algorithm POCS parallel wil converge to a point such that the sum of the squares of the distances to the sets is minimized. This case is geometrically represented by Figure 3.

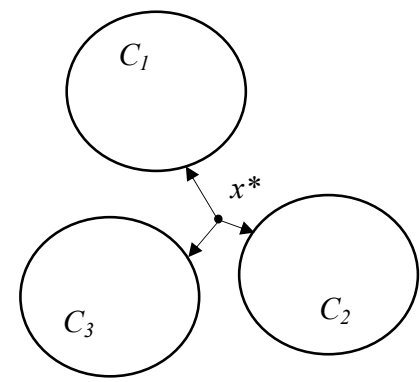

Figure 3: Algorithm behavior of a parallel POCS when there is no intersection of the convex sets

\subsection{Convex Restriction Sets}

The image reconstruction linear model can be described by Eq (5), where $y$ represents the projection vector data, $f$ is the original image vector, $H$ is the projection matrix, and $n$ is the noise vector.

$y=H f+n$

Eq. (5) describes a linear system of equations, where each component of $y$ describes a linear equation, which can be represented by a hyperplane. Each hyperplane is a convex set which represents a restriction of the POCS method, and the solution of the linear system can be found through the POCS method. The projector for $i$ th component of $y$ is given by Eq. (6), where $\hat{f}$ is any estimation of $f$.

$P_{C_{i}}=\hat{f}-\frac{\left\langle H_{i}, \hat{f}\right\rangle-y_{i}}{\left\|H_{i}\right\|^{2}} H_{i}$

Besides the restrictions sets expressed by Eq. (5), it is possible to consider the a priori knowledge about the image to be reconstructed. Such restrictions must be 
expressed in a set form. Eq. (7) describes the finite support set, where $S$ is a Hibert Space, $h$ is the obtained image and $\Omega$ is the region that describes the support.

$C_{F S}=\{h: h \in S$ and $h(x, y)=0$ for $(x, y) \notin \Omega\}$

The projection operator of the set defined by Eq. (7) is shown in Eq. (8).

$P_{F S}=\left\{\begin{array}{cc}h(i), & \text { if } i \in \Omega \\ 0, & \text { if } i \notin \Omega\end{array}\right.$

Eq. (9) describes the non-negative set.

$C_{+}=\{h: h \in S$ and $h(i) \geq 0 \forall i\}$

$P_{+}=\left\{\begin{array}{cc}h(i), & \text { if } h(i) \geq 0 \\ 0, & \text { otherwise }\end{array}\right.$ (10).

The rule projection on the $C_{+}$is described by Eq.

\section{Poisson Noise Filtering}

The Poisson noise comes from the discrete nature of radiation that characterizes the counting process of particles in the projections set. The reconstruction method is performed by first filtering the noisy projection and subsequently reconstructing the image through parallel POCS method.

\subsection{The Anscombe Transform}

The Anscombe Transform (AT) [5] transforms the signal-dependent Poisson noise into an approximately Gaussian, additive, signal-independent noise with zero mean and unit variance.

The AT on $y_{i}$ is given by Eq. (11), where $z_{i}$ can be represented by an additive model as described by Eq. (12).

$$
\begin{aligned}
& z_{i}=2 \sqrt{y_{i}+\frac{3}{8}} \\
& z_{i}=2 \sqrt{g_{i}+\frac{1}{8}}+v_{i}=s_{i}+v_{i}
\end{aligned}
$$

In Eq. (12) $v_{i}$ is an additive noise that is approximately independent of $s_{i}$, and $g_{i}$ is the signal without Poisson noise.

The inverse AT on $z_{i}$ is given by Eq. (13). $\hat{g}_{i}=\frac{1}{4} z_{i}^{2}-\frac{1}{8}$

\subsection{Wavelets and Denoising}

The joint time-frequency analysis of the wavelet basis efficiently captures non-stationary signal features. The discrete wavelet transform (DWT) represents a signal in terms of shifted versions of a low-pass scaling function $\phi$ and shifted and dilated versions of a prototype band pass wavelet function $\psi$.

$\psi_{j, k}(t)=2^{j / 2} \psi\left(2^{j} t-k\right)$

$\phi_{j, k}(t)=2^{j / 2} \phi\left(2^{j} t-k\right)$

Eqs. (14) and. (15) form an orthonormal basis for special choices of $\phi$ and $\psi$, where $j, k \in Z$.

The signal $y(t)$ can be represented by Eq (16), and the components $u_{j, k}$ and $w_{j, k}$ represented by Eqs. (17) and $\mathrm{Eq}(18)$.

$$
\begin{aligned}
& y(t)=\sum_{k} u_{j_{0}, k} \phi_{j_{0}, k}(t)+\sum_{j=j_{0}}^{J} \sum_{k} w_{j, k} \psi_{j, k}(t) \\
& u_{j, k}=\int y(t) \phi_{k, k}^{*}(t) \partial t \\
& w_{j, k}=\int y(t) \psi_{k, k}^{*}(t) \partial t
\end{aligned}
$$

The parameter $J$ in Eq. (16) controls the resolution in the wavelet representation.

The noise can be filtered in different wavelets scales, through a threshold that can be chosen depending of the wavelet scale.

Another possibility is to filter the noise in the wavelet domain by using a pointwise Wiener filter, considering a window in a given scale. This is the approach that was used in this paper.

\subsection{Local Wiener Filter}

Assuming that the signal components in a wavelet space are not correlated, and the noise is white, with zero mean and unitary variance, the pointwise Wiener filter can be represented by Eq. (19).

$$
\hat{s}_{i}=E\left\{s_{i}\right\}+\frac{\sigma_{s_{i}}^{2}}{\sigma_{s_{i}}^{2}+1}\left(z_{i}-E\left\{s_{i}\right\}\right)
$$


The expected value $E\left\{s_{i}\right\}$ and the variance $\sigma_{s_{i}}^{2}$ are estimated on the neighborhood of coefficient $z_{i}$. Taking into account the zero mean of the noise, the expected value of the signal can be estimated by the sample mean of the noisy signal over the neighborhood. The variance is also estimated by the sample variance of the noisy signal and subtracted by one (the variance of the additive noise). If the result is negative, the estimated signal is given by the sample mean.

\section{Tomographic Reconstruction Method}

The method consists, initially, of filtering the noisy projections. Since the noise can be modeled as Poisson noise under low counts, the AT can be used, and an additive noise model is obtained. Following it, the DWT is performed, allowing noise suppression that can be performed in different scales. The mean value is null and the variance is the same in every scale. By using the pointwise Wiener filter we obtain an estimate of the signal and, by performing an Inverse Discrete Wavelet Transform (IDWT) followed by the Inverse Anscombe Transformation, we obtain an estimation of the noise free projections. This previous estimation procedure tighten the observations restrictions of the POCS tomographic reconstruction method, improving the quality of the solution $[6,7,8]$.

After the estimation of the projections, the parallel POCS algorithm is applied for the tomographic reconstruction of the image, in accordance with Eq. (3) and by using the restriction sets described by Eq. (5), and Eq. (7), and Eq. (9).

\section{Numerical Results}

The reconstruction of three phantoms (see Figure 1) was performed. The images reconstructed with the highest exposure (twenty seconds per ray) of gammaray were considered the ideal image and the images reconstructed with three seconds per ray are the noisy images that we want to reconstruct.

The ideal reconstructed images are shown in Figure 4. In this case the reconstruction was performed with the parallel POCS algorithm. (a)

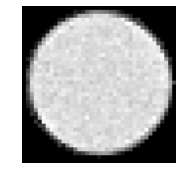

(b)

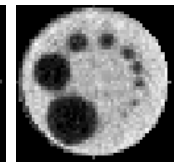

(c)

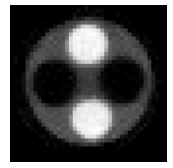

Figure 4: Ideal Phantom Images: (a) homogeneous; (b) asymmetrical; (c) symmetrical
Figure 5 shows the reconstructed phantoms without filtering the projections. (a)

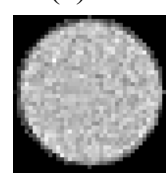

(b)

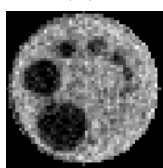

(c)

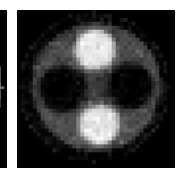

Figure 5: Non-Filtered Images: (a) homogeneous; (b) asymmetrical; (c) symmetrical

Figure 6 shows the phantoms reconstructed by the hybrid estimation theoretic - POCS method that was proposed. (a)

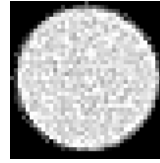

(b)

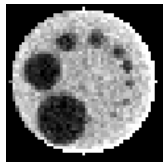

(c)

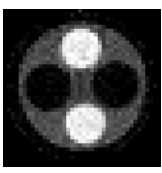

Figure 6: Filtered Images:

(a) homogeneous; (b) asymmetrical; (c) symmetrical

A performance measure was computed based on the improvement in signal-to-noise ratio (ISNR) in decibel $(\mathrm{dB})$ which is defined by Eq. (20), where $f_{j}$ is the $j$ th element of the ideal image, $\hat{f}_{j}$ is the $j$ th element of the image reconstructed by the method proposed, and $g_{j}$ is the $j$ th element of the image reconstructed without filtering the projections.

$$
I S N R=10 \log \frac{\sum_{j}\left(f_{j}-g_{j}\right)^{2}}{\sum_{j}\left(f_{j}-\hat{f}_{j}\right)}
$$

For the reconstruction of the asymmetrical phantom, the ISNR was $5.57 \mathrm{~dB}$. For the homogeneous phantom the ISNR was $2.38 \mathrm{~dB}$, and for the symmetrical phantom the ISNR was $3.14 \mathrm{~dB}$.

\section{Conclusions}

The hybrid estimation-theoretic-POCS technique using Anscombe Transformation, Discrete Wavelet Transform and pointwise Wiener Filter for the filtering of the projections and parallel POCS for the image reconstruction displayed a better result than a pure parallel POCS approach in ISNR and visual terms. This is due to the fact that the previous method uses an 
estimation procedure that further reduces the restriction sets of the POCS procedure that describe the measurements under noisy conditions.

The addition of the estimation-theoretic procedure on the projections represents a very low computational cost, compared to the parallel POCS reconstruction technique, which is an iterative procedure. Therefore, the proposed method has a lower cost-benefit measure, compared to the pure POCS procedure.

The proposed method can be improved, since we used the Haar basis for the Discrete Wavelet Transform. We believe that a better result can be obtained by trying other wavelet bases.

\section{Acknowledgement}

This work was partially supported by FAPESP Thematic Project 2002/07153-2. The authors want to thank Dr. Paulo Estevão Cruvinel for providing the phantom images.

\section{References}

[1] N.D.A. Mascarenhas, S.S.L Santos, and P.E. Cruvinel, "The Use of MAP Techniques in the Tomographic Reconstruction of Poisson Noise Corrupted Images", IX Brazilian Symposium on Computer Graphics and Image Processing, Caxambu, Brazil, pp 197-204, 1996

[2] N.D.A. Mascarenhas, S.S. Furuie, and L.S. Portal, "Global Projection Estimation Methods for the Tomographic Reconstruction of Images with Poisson Noise", IEEE Transactions on Nuclear Science N.40, pp 2008-2013, 1993
[3] F.V. Salina, N.D.A. Mascarenhas, and P.E. Cruvinel "A Comparison of POCS Algorithms for Tomographic Reconstruction Under Noise and Limited View", XV Brazilian Symposium on Computer Graphics and Image Processing, Campinas, Brazil, pp 342-346, 2002.

[4] P.L. Combettes, "The Foundations of Set Theoretic Estimation”, Proc. IEEE, v. 81 n. 2, pp 182-208, 1993.

[5] F.J. Anscombe, "The Transformation of Poisson, Binomial and Negative-Binomial data", Biometrika v.15), pp 246-254. 1948

[6] C. Charles and J.P. Rasson, "Wavelet Denoising of Poisson-Distributed Data and Applications", Computational Statistics \& Data Anlysis, pp 139-148, 2003.

[7] M. R. P. Homem, N. D. A. Mascarenhas, L. F. Costa, and C. Preza, "Biological Image Restoration in Optical-Sectioning Microscopy Using Prototype Image Constraints", Real-Time Imaging 8, pp 475-490, 2002

[8] M. Kazubek, "Wavelet Domain Image Denoising by Thresholding and Wiener Filtering", IEEE Signal Processing Letters, v. 10, n 11, pp 324-326, 2003

[9] D. C. Youla, H. Webb. "Image restoration by the method of convex projections: part 1 - Theory", IEEE Trans. Med. Imaging, MI-1, pp 81-94, Oct, 1982 